\title{
Psychological First Aid (PFA) in Improving Staff Confidence in an Eye Hospital during Times of Uncertainty from Ensuing Covid19 Pandemic
}

\author{
Kaushik Murali ${ }^{*}$, Gokul Ratakonda ${ }^{2}$ and Geeta Fulari ${ }^{3}$ \\ ${ }^{1}$ President, Medical Administration, Sankara Eye Foundation, India \\ ${ }^{2}$ Clinical Psychologist and Research Associate, Sankara Eye Foundation, India \\ ${ }^{3}$ Manager - Quality Assurance, Sankara Eye Foundation, India \\ *Corresponding Author: Kaushik Murali, President, Medical Administration, \\ Sankara Eye Foundation, India.
}

Received: April 06, 2021

Published: April 30, 2021

(C) All rights are reserved by Kaushik Murali., et al.

\section{Abstract}

Purpose: To study the effect of using principles of Psychological First Aid (PFA) in improving healthcare worker (staff) confidence in an eye hospital during times of uncertainty from ensuing COVID19 pandemic.

Methods: Patient Health Questionnaire was designed to gather data about the impact of current pandemic on doctors and staff. Data was also gathered through interviewing the staff and the doctors. To address all the concerns, 5 components of Psychological First Aid (PFA) in communication were used, and also in the provisions made at the workplace to help alleviate this impact. PFA is an initial disaster response intervention with the goal to promote safety, stabilize survivors of disasters and connect individuals to help and provide resources. Staff were re-interviewed to assess their confidence levels. Campaign was created to look at other aspects of routine hospital functioning that could impact safety and outcomes beyond COVID19 and report to the Quality Team.

Results: The mean PHQ before interventions was 3.98. Post intervention and return to work, the PHQ score in doctors and staff was only 0.94 . Survey showed absolutely no signs of any psychological distress amongst staff. Targeted trainings showed $94 \%$ response rate. 3\% staff opted for sabbatical which was taken as a surrogate for safety. A random survey of 29 staff showed that $48 \%$ were confident of all tasks adhering to COVID19 protocols, which increased to $96.5 \%$ in 3 months.

Discussion: Motivation of healthcare workers was seen to be beyond just financial aspects. There was a strong sense of belonging and duty as identified in our survey. This increases the onus on organisations towards addressing the concerns of healthcare workers providing psychological support. PFA is a proven tool that can help reduce distress in a time of fear, anxiety and uncertainty. Though we did not implement the PFA in the manner it is used typically in natural disasters for individuals, implementing a programme, looking at the principles of - feeling safe, connected, efficacious, calm and hopeful for the cohort of health workers, brought down the distress as measured by the PHQ-9 questionnaire from 3.2 to 0.94 and seems to have a value even beyond the pandemic.

Conclusion: Implementing targeted interventions based on the principles of psychological first aid can help reduce psychological distress among health care workers in times of uncertainty in an eye hospital.

Keywords: COVID-19; PFA (Psychological First Aid); Psychological Distress; Pandemic; PHQ (Patient Health Questionnaire); Counselling; SanQALP - Sankara Quality Assurance and Learning Programme 


\section{Abbreviations}

COVID: Coronavirus Disease; PHQ: Patient Health Questionnaire; PFA: Psychological First Aid; PPE: Personal Protective Equipment

\section{Introduction}

Sankara Eye Foundation, India is a network of 11 eye hospitals across India, delivering eye care with a social impact with an aim to achieve our vision of freedom from preventable and curable blindness for all. Healthcare workers fighting against Coronavirus disease 2019 (COVID-19) pandemic are under tremendous pressure, which puts them at an increased risk of developing psychological problems [1].

The World Health Organisation has defined Psychological First Aid as 'humane, supportive response to a fellow human being who is suffering and who may need support' [2]. Originally envisaged for providing immediate care at times of emergency, the current pandemic has shown distress similar to major incidents [3]. PFA has been devised as an approach and a tool, the key outcomes of PFA, such as feeling safe, connected, efficacious, calm and hopeful, are reported as effective in helping long-term recovery following a crisis, even when delivered by individuals without professional mental health training [4].

A survey among Indian Ophthalmologists indicated that they perceived themselves to be at a higher risk of contracting COVID-19 as compared to other specialties. The survey conducted during the first lockdown also showed a lack of clarity among clinicians about how to proceed after the resumption of routine services [5]. Another survey among trainees showed that majority of the ophthalmology trainees across the country felt that COVID-19 lockdown adversely affected their learning, especially surgical training [6]. Risk factors for mental health include overwhelming situations, social disruption of daily life, feeling vulnerable, at risk of being infected, fear of transmitting the disease to families, and loved ones [7].

Our Clinical Psychology team (Mind Matters) at Sankara Eye Hospital Bangalore conducted One to one interviews with the doctors and staff using a pre designed questionnaire to understand any concerns. Along with the responses received, other aspects like body language and expressions were considered. 'I feel future is uncertain', 'I am not sure if I should be really coming to work', 'Given a chance I would love to be with my kids at home till this pandemic ends', these have been some of the responses given by the ophthal- mologists. However, there were also some staff who said 'It's my moral responsibility to come to work and provide my services', 'Rather than sitting at home, I love to come to work inspite of this pandemic' have also been the responses given by some staff.

Recognising the distress among our stakeholders of staff, trainees and patients we looked at implementing programme that specifically fulfiled facets of the PFA as relevant to an eye hospital environment. The objective was to improve confidence amongst our staff and doctors and thereby improve our patient care services.

\section{Methods}

Design thinking principles were applied to ideate and arrive at possible avenues of intervention that could be aligned to each principle of Psychological first aid Principles

i) Create a sense of safety: SPARK which is an acronym for SSocial distancing, P-Use of Personal Protective Equipment (PPEs), A -Adherence to guidelines, R-Regulate self (isolate), $\mathrm{K}$ - Keep hands clean, was championed through the SanQALP (Sankara Quality Assurance and Learning Programme. SPARK was used as a tool to educate patients and staff on what to expect in daily functioning. The layout was restructured to ensure social distancing at all levels. Floor markings were made to help queue management. PPEs were sourced to enable task specific protection. We worked with 3D printing startups to procure face shields and UV-C sterilisers. Standard Operating procedures were drafted and the through multiple trainings through webinars and small group interactions ensured staff were empowered with all the knowledge and support they needed. Regular monitoring and internal audits were conducted to ensure implementation of these policies. Screening at entrance for temperature, an automated self declaration by staff on their health helped isolate and contain. Through the modified set of solutions, it was ensured that staff and patients were guided and educated at every step on maintaining their personal hygiene.

Online lectures combined with several educational videos were uploaded on YouTube so that they could be easily referenced. Teams were formed to track guidelines and advisories.

ii) Create calm: To orient emotionally overwhelmed an 'Assent policy' and sabbatical was offered to those with pre-existing conditions or those who had dependants at home they thought were at high risk. With the help of an Inhouse qualified psy- 
chologist, one to one sessions were provided to the staff who felt the need to help with their anxiety. The senior management interacted with departmental staff in open houses to address their concerns and orient them towards the safety norms. A video message from our founders was recorded and shared with all staff to allay apprehensions.

iii) Create self and collective efficacy: A COVID Task Force comprising senior colleagues was created with strategic Teams formed to look at specific areas like PPE, disinfection protocols, patient care departments, rostering where they tracked guidelines and advisories and ensured almost continuous updation of our protocols. Patient journey in the hospital was relooked to ensure speeding up of the whole process. With the preoccupation due to the pandemic, we realised that routine drills cannot be overlooked and a campaign 'Be Ready' was launched. This included ensuring confirmation of fire safety including Code red mock drills, ambulance readiness, checking crashcarts, compliance to COVID19 safety policies, proper Biomedical waste disposal, confirming non availability of expiry drugs, water quality checks, etc.

iv) Create connection: Our trainees were most despondent. A lecture series was launched with a lecture at noon every day for 36 days continuously. We also ensured engagement pro- moting social interaction through regular online meetings that were without an agenda. A video that brought together aspects of the hospital that staff found dear and listed through a poll at the hospital was also created and shared with all staff. Continuous trainings on safety measures and online lectures combined with several educational videos were uploaded on YouTube so that they could easily be referenced and gave them a sense of purpose.

v) Create hope: A continuous pipeline of communication to focus on what is being done was promoted using social media tools like WhatsApp and the human resource management (HRM) portal, a 'Corona kavach' policy was taken and all hospitals signed up MOUs with critical care facilities to prioritise treatment for staff and their families if they required hospitalisation. Staff with COVID were encouraged to communicate with others, with a doctor even handling a seminar from the hospital while being treated for COVID19, which helped others gain confidence. To bring in positivity about the state of mind, a video was created and disseminated to every staff (https://youtu.be/puRo0S66a9A).

A PHQ (Patient Health Questionnaire) for doctors and staff to gather data related to Psychological impact on them due to the pandemic situation was administered.

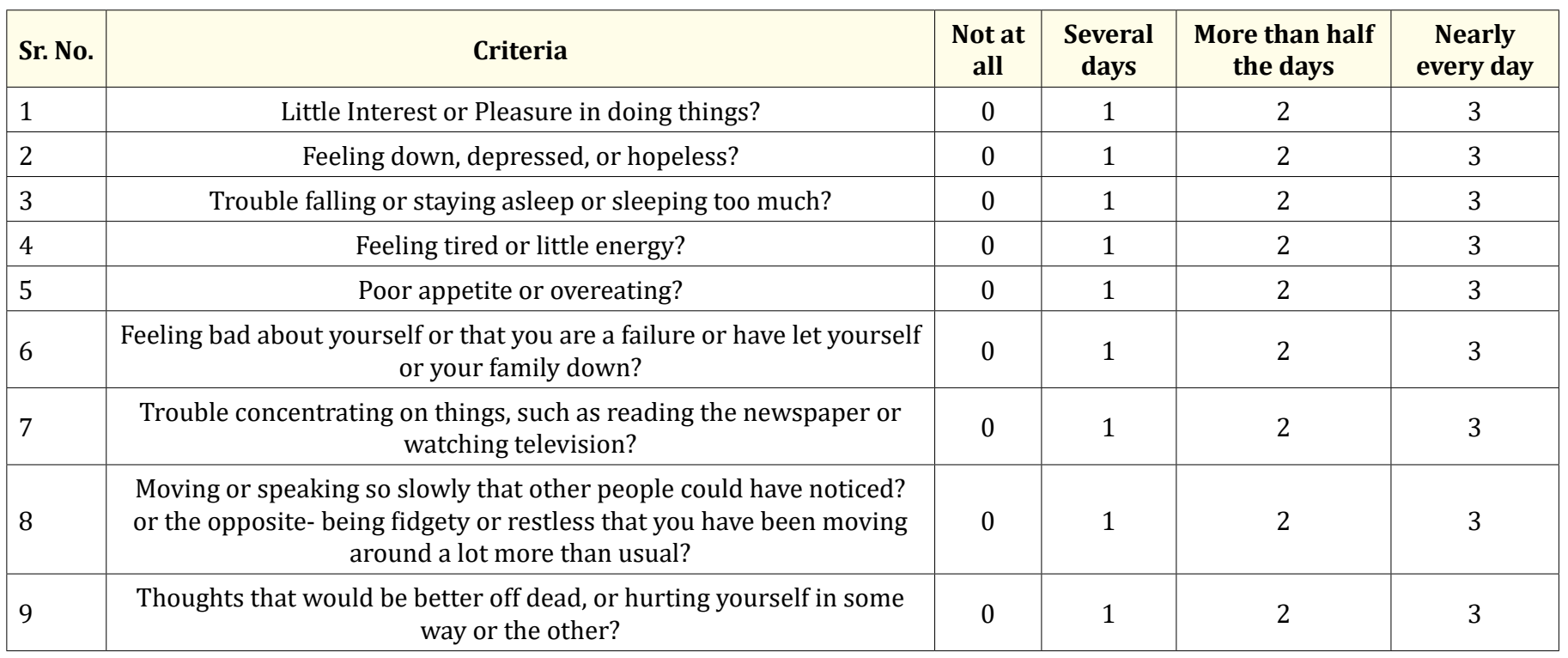

Table 1: Patient health questionnaire (PHQ 9).

Insights from patients and staff were collated and a Word Cloud generated using an online site (https://wordart.com/). 
29 staff across cadres were identified in a cross sectional survey on a single day to understand their confidence levels in handling tasks related to their role based on COVID19 protocols and guidelines. The same staff were re-interviewed in July 2020 on their confidence levels.

Campaign was created to look at other aspects of routine hospital functioning that could impact safety and outcomes. Hospitals were asked to share an audit report of various tasks that were completed and report to the Quality Team. These included - Quantity of fire extinguishers identified that were due for refilling, Quantity of fire extinguishers that had crossed refilling due date, Number of times Non-compliance towards BMWD was found, Quantity of expired medications found, Quantity of near expiry medications found, No. of Temperature monitoring checklists that were not updated, Number of oxygen cylinders found with insufficient cylinder pressure, Number of crashcart checklists not updated, Incidences of PPE not disposed in correct bins. Those opting for Sabbatical was taken as a surrogate for staff feeling safe at work.

Data was analysed using Microsoft Excel.

\section{Results}

Targeted trainings showed 94\% response rate. 97\% of all staff continued to work through the year. 3\% staff opted for sabbatical and all of them returned to work after the initial phase of lockdown and the first wave. Weekly review of SOPs was done through lockdown.

A word cloud analysis (Figure 1) of inputs from staff showed a high level of sense of duty, followed by financial needs and a yearning to learn being the main criteria that encourages staff to work despite the COVID19 lockdown.

A similar analysis of patients who had reported to the OPD in what they expected when they considered an eye care facility, put a sense of being cared for even higher than safety and hygiene. This was attributed to them assuming that hospitals would have strong infection control protocols.

29 staff interviewed, in the preintervention phase (May 2020), it was found that 12 staff were not confident to carry out their tasks in the background of the prevailing COVID19 pandemic, 3 could performs their tasks but under guidance, 14 (48.2\%) staff were confident to carry out their tasks. The same staff on implementation of the PFA and training, in the post implementation phase (July 2020) out of the 29 staff, it was found that 1 staff was able to carry out their tasks under guidance, 28 (96,5\%) were now confident enough to carry out their tasks independently.

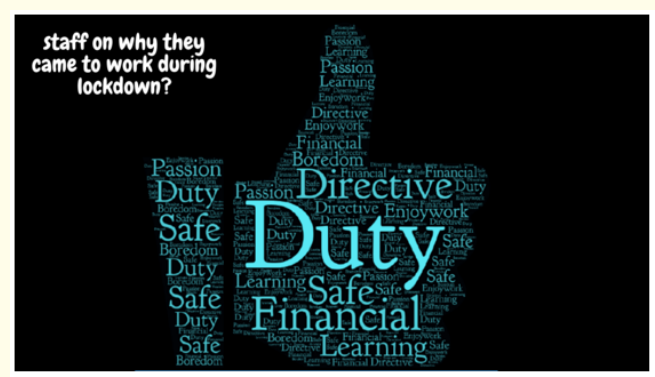

Figure 1: Word cloud of why staff were coming to work during lockdown.

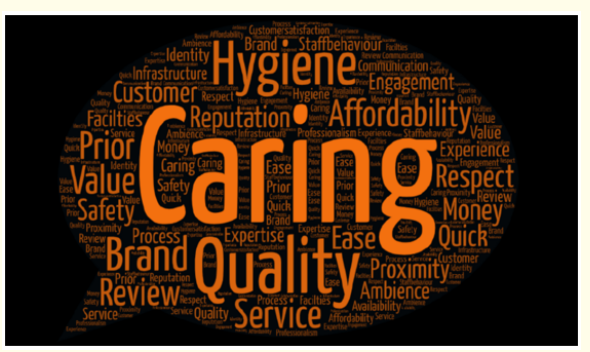

Figure 2: Word cloud of patient insights explaining what makes patients visit the hospital despite the ongoing pandemic.

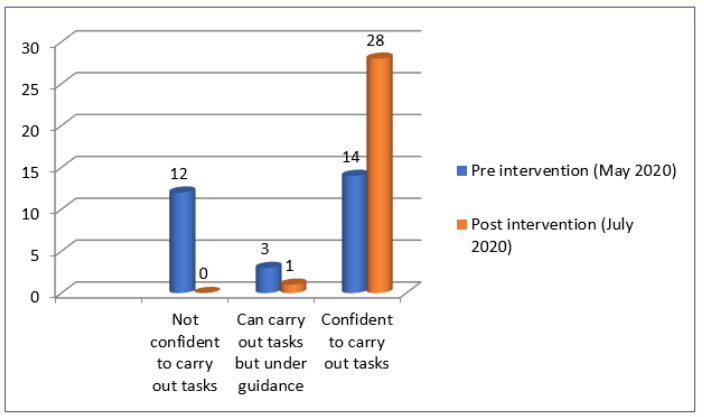

Figure 3: Graphical presentation of confidence level of all staff to work with COVID19 guidelines. 
Psychological First Aid (PFA) in Improving Staff Confidence in an Eye Hospital during Times of Uncertainty from Ensuing Covid19 Pandemic

\begin{tabular}{|l|c|c|c|c|c|c|c|c|c|c|c|c|c|c|}
\hline Sr. & Task & AND & BLR & CBE & CBE RSP & GNT & IND & JPR & KK & KNP & LDH & SMG \\
\cline { 2 - 11 } No. & \multicolumn{7}{|c|}{ Number of times non-compliance was observed } \\
\hline 1 & $\begin{array}{c}\text { Quantity of fire extinguishers identified that } \\
\text { were due for refilling }\end{array}$ & 22 & 1 & 43 & 5 & 15 & 0 & 0 & 3 & 2 & 0 & 0 \\
\hline 2 & $\begin{array}{c}\text { Quantity of fire extinguishers that had crossed } \\
\text { refilling due date }\end{array}$ & 0 & 0 & 43 & 4 & 50 & 0 & 0 & 3 & 0 & 0 & 0 \\
\hline 3 & $\begin{array}{c}\text { Number of times Non-compliance towards } \\
\text { BMWD was found }\end{array}$ & 0 & 5 & 0 & 1 & 1 & 1 & 0 & 5 & 0 & 2 & 5 \\
\hline 4 & Quantity of expired \medications found & 0 & 7 & 0 & 1 & 0 & 0 & 0 & 0 & 50 & 0 & 55 \\
\hline 5 & Quantity of near expiry medications found & 47 & 36 & 0 & 2 & 1 & 0 & 0 & 417 & 99 & 0 & 20 \\
\hline 6 & $\begin{array}{c}\text { No. of Temperature monitoring checklists that } \\
\text { were not updated }\end{array}$ & 0 & 1 & 0 & 0 & 0 & 0 & 0 & 0 & 0 & 0 & 2 \\
\hline 7 & $\begin{array}{c}\text { Number of oxygen cylinders found with insuf- } \\
\text { ficient cylinder pressure }\end{array}$ & 3 & 0 & 0 & 0 & 0 & 0 & 0 & 1 & 0 & 1 & 0 \\
\hline 8 & Number of crashcart checklists not updated & 0 & 1 & 0 & 0 & 0 & 2 & 0 & 0 & 0 & 1 & 1 \\
\hline 9 & Incidences of PPE not disposed in correct bins & 0 & 4 & 0 & 6 & 1 & 0 & 0 & 2 & 0 & 0 & 1 \\
\hline
\end{tabular}

Table 2: Shortcomings found after conducting campaign activities in every unit.

The mean PHQ before interventions was 3.2. Post two weeks after interventions and return to work, the PHQ score in our doctors and staff was only 0.94 . Results showed absolutely no signs of any psychological distress amongst staff.

\section{Discussion}

HCWs are at risk for developing physical and mental health consequences due to their role in providing care to patients with COVID-19. Implementation of effective strategies may help reduce the burden of health consequences: the adequate provision and training on the use of personal protective equipment, strict infection control practices, shorter shift length, and provision of mental health and support services [7]. Better organizational support has also been found to allay fears in medical staff [8].

Safety from infection of their family, friends, and colleagues; fear and uncertainty and possible stigmatization have been seen to result in high levels of stress, anxiety, and depression symptoms, among front line health workers in various studies [9].

Motivation of healthcare workers to work in difficult situations, where they put themselves at risk was seen to be beyond just financial aspects. There was a strong sense of belonging and duty as identified in our survey. This increases the onus on organisations towards addressing the concerns of healthcare workers providing psychological support. Also, with patients expecting to be cared for, unless the staff are in a mind-set of calm, this would be an expectation difficult to fulfil.
Healthcare workers have a much higher risk of psychological problems (E.g. anxiety, depression and insomnia) during the epidemic [10]. This may be related to the higher risk of infection on account of being exposed to patients with COVID-19 and tedious work involved in caring for them and reminds us of the importance of providing psychological support to healthcare workers during the pandemic [1]. A study showed that strikingly high proportion of ophthalmologists are psychologically affected, with PHQ score: 3.98 and may require personalized mental health care [11].

Organisations to relook at their staff and patients and prioritise on aspects important to keep them safe and engaged. It is also important to look at basic practices that may impact patient and staff safety and outcomes. The lockdowns and the focus on COVID19 resulted in possible oversight in various areas from drugs nearing expiry to fire extinguishers needing upkeep. It is imperative that the focus remains on overall quality of care and safety despite trying conditions.

As organisations plan for uncertainties, PFA is a proven tool that can help reduce distress in a time of fear, anxiety and uncertainty. It has been delivered by non-psychologists as an emergency intervention [12]. Though we did not implement the PFA in the manner it is used typically in natural disasters for individuals, implementing a programme looking at the principles for the cohort of health workers seems to have a value even beyond the pandemic.

Comprehensive measures to assess and reduce the psychological stress of hospital workers are needed. Symptoms should be 
monitored with vigilance, and further intervention should be provided, if necessary [13]. PHQ (Patient Health Questionnaire) is a screening tool for mental health status and can give insights on the intensity of psychological distress. Serial measurements can aid in understanding the evolving status of individuals in an objective manner and help plan interventions.

This showed that it is necessary to promote monitoring the health status, including mental health of health workers during moments of crisis [14].

\section{Conclusion}

COVID19 has brought lot of uncertainty in everyone's lives. It has greatly affected the work life of healthcare workers across the globe. Implementing targeted interventions based on the principles of psychological first aid can help reduce psychological distress in times of uncertainty.

\section{Financial Support and Sponsorship}

Nil.

\section{Conflicts of Interest}

There are no conflicts of interest.

\section{Bibliography}

1. Que J., et al. "Psychological impact of the COVID-19 pandemic on healthcare workers: a cross-sectional study in China". General Psychiatry 33.3 (2020): e100259.

2. World Health Organization. "War Trauma Foundation, World Vision International. Psychological First Aid: Guide for Field Workers". World Health Organization: Geneva (2011).

3. Minihan E., et al. "COVID-19, mental health and psychological first aid”. Irish Journal of Psychological Medicine 37.4 (2020): 259-263.

4. Fox JH., et al. "The effectiveness of psychological first aid as a disaster intervention tool: research analysis of peer-reviewed literature from 1990-2010". Disaster Medicine and Public Health Preparedness 6.3 (2012): 247-252.

5. Nair AG., et al. "Effect of COVID-19 related lockdown on ophthalmic practice and patient care in India: Results of a survey". Indian Journal of Ophthalmology 68.5 (2020): 725-730.
6. Mishra D., et al. "The impact of COVID-19 related lockdown on ophthalmology training programs in India - Outcomes of a survey". Indian Journal of Ophthalmology 68.6 (2020): 9991004.

7. Shaukat N., et al. "Physical and mental health impacts of COVID-19 on healthcare workers: a scoping review". The International Journal of Emergency Medicine 13 (2020): 40.

8. Philip J and Cherian V. "Factors Affecting the Psychological Well-being of Health Care Workers During an Epidemic: A Thematic Review". Indian Journal of Psychological Medicine 42.4 (2020): 323-333.

9. Lai J., et al. "Factors associated with mental health outcomes among health care workers exposed to coronavirus disease 2019". JAMA Network Open (2020).

10. McAlonan GM., et al. "Immediate and sustained psychological impact of an emerging infectious disease outbreak on health care workers". The Canadian Journal of Psychiatry 52.4 (2007): 241-247.

11. Khanna RC., et al. "Psychological impact of COVID-19 on ophthalmologists-in-training and practising ophthalmologists in India”. Indian Journal of Ophthalmology 68 (2020): 994-998.

12. Minihan E., et al. "COVID-19, mental health and psychological first aid". Irish Journal of Psychological Medicine 37.4 (2020): 259-263.

13. Yang S., et al. "Psychological impact of COVID-19 on hospital workers in nursing care hospitals". Nursing Open (2020): 1-6.

14. Giusti EM., et al. "The Psychological Impact of the COVID-19 Outbreak on Health Professionals: A Cross-Sectional Study". Frontiers in Psychology 11 (2020): 1684.

\section{Assets from publication with us}

- Prompt Acknowledgement after receiving the article

- Thorough Double blinded peer review

- Rapid Publication

- Issue of Publication Certificate

- High visibility of your Published work

Website: www.actascientific.com/

Submit Article: www.actascientific.com/submission.php

Email us: editor@actascientific.com

Contact us: +919182824667 